\title{
Fluorescent Pseudomonads as Plant Growth Promoting Rhizobacteria and Their Siderophoregenesis
}

\author{
${ }^{1}$ Bholay A. D., Jadhav Priyanka.U., ${ }^{2}$ Borkhataria B. V.,Mayuri V. Dhalkari. \\ ${ }^{l}$ P.G. Dept. of Microbiology, K.T.H.M. College, Nashik, M.S, India. \\ ${ }^{2}$ Curie Manavata Cancer Centre, Nashik, M.S., India.
}

\begin{abstract}
Two fluorescent Pseudomonads, Pseudomonas fluorescens NCIM 5164 and Pseudomonas aeruginosa NCIM 2036 produced siderophores under iron limiting conditions. These Pseudomonas spps. were screened for siderophore production by three different tests commonly used to detect siderophores. Quantitative estimation of siderophores was done by the Chrome Azurol S assay (CAS), the universal test independent of siderophore type. P. fluorescens and P. aeruginosa produced $88 \%$ and $83 \%$ siderophore units respectively. The type of siderophore was detected by chemical as well as spectrophotometric assay. Pseudomonas fluorescens was found to produce hydroxamate type of siderophore and Pseudomonas aeruginosa produced both the hydroxamate (Pyoverdin) and phenol-catecholate (Pyochelin) siderophores. Evaluation study of siderophore showed that maximum siderophore production was obtained on succinate medium at $\mathrm{pH}$ 7.0. Both the Pseudomonas spps. were further tested as seed inoculants and found to be very effective in seed germination and plant growth promotion of Triticum aestivum and Apios americana plants under pot culture conditions.
\end{abstract}

KeyWords - CAS, Catecholate, Hydroxamate, Pyoverdin, Pyochelin, Siderophores.

\section{Introduction}

Rhizosphere is a dynamic environment which harbours diverse group of microbes. The bacteria which directly or indirectly stimulate plant growth have been referred to as plant growth promoting rhizobacteria (PGPR). PGPR promote growth of several annual crops by increasing uptake of nitrogen, iron (through siderophores), phosphorus, synthesis of phytohormones and by controlling plant diseases [1].

Iron is an essential trace element for living organisms. Because of its redox activities and ability to form co-ordination compounds with variety of ligands, it is a constituent of a large number of vital enzymes. Despite being most abundant elements in earth's crust, the availability of iron is limited by the very low solubility of $\mathrm{Fe}^{3+}\left(10^{-17}\right)$ predominant state of iron in aqueous, non-acidic, oxygenated environments and accumulates in mineral phases such as iron hydroxides as rust, hence cannot be utilized by organisms. In response microorganisms have developed a strategy for acquiring iron, which includes synthesis and utilization of siderophores.

A Siderophore (Greek for iron carrier) is a low molecular weight (500-1000 daltons), high affinity ferric iron chelating compound secreted by organisms. Siderophores scavenge iron from mineral phases by formation of soluble $\mathrm{Fe}^{3+}$ complexes that can be taken up by energy dependent membrane transport mechanism and thus bind it and transport it to plants or bacterial cells. Siderophores which are wide spread among bacteria are of three types based on chemical nature of their co-ordination sites. Hydroxamate siderophore possesses $\mathrm{N}$ hydroxylated amide bonds as co-ordination sites, catecholates co-ordinate iron with catecholate hydroxyl group and carboxylates co-ordinate iron with carboxyl and hydroxyl groups.

Pseudomonas spps. have been known for their siderophore production for many years and therefore many reports on the isolation and characterization of their siderophores have been published [2,3]. The importance of siderophore extends their applications in agriculture, biotechnology and medicine. Siderophore produced by Pseudomonas spp. have been employed efficiently as biocontrol agents against certain soil-borne plant pathogens.

Pseudomonas aeruginosa produces an extracellular compound with yellowish green fluorescence, called pyoverdin $(\mathrm{Pvd})$, which functions as a siderophore. The production of pyoverdin, formerly called fluorescein, is concomitant with the production of another siderophore, pyochelin $[4,5]$.

Pyoverdin is a more complex molecule (approximately 1,500 daltons) consisting of 8hydroxyquinoline derivative, which forms a fluorescent chromophore and an octapeptide which contains two residues of $\mathrm{N}_{5}$-hydroxyornithine [6]. The hydroxamate groups formed by these two residues participate in the binding of iron together with the catecholate group of the chromophore. Pvd is a fluorescent dihydroxyquinoline derivative connected to a small peptide and contains hydroxamate and catecholate residues to chelate ferric ion, Fe (III). The hydroxamate siderophore pyoverdin has a greater affinity for Fe (III) than has Pyochelin [7]. Pyoverdin is highly water-soluble molecule and a powerful chelator of ferric iron, which is bound with a stoichiometry of 1:1 and a stability constant of approximately $10^{24} \mathrm{M}^{-1}$ at neutral $\mathrm{pH}$ [8]. Both features favor $\mathrm{Fe}$ 
(III) binding to pyoverdin with respect to Pyochelin [9]. Pyochelin is an unusual siderophore in that it is poorly water-soluble, low-molecular weight (325 daltons) [10] which binds Fe (III) with a stoichiometry of two molecules per iron atom and a remarkably low stability constant of approximately $2 \times 10^{5} \mathrm{M}^{-2}$ [11].

The present study has been undertaken for production, detection and estimation of siderophores produced by two Pseudomonas spps. and their role in growth promotion of Triticum aestivum and Apios americana plants under pot culture conditions.

\section{Materials And Methodology}

\subsection{Inoculum Preparation}

A loopful culture of Pseudomonas fluorescens and Pseudomonas aeruginosa from Nutrient agar slants were separately inoculated in $100 \mathrm{ml}$ of Kings B medium. These flasks were incubated for 24 hours at $28^{\circ} \mathrm{C}$ with constant shaking at $120 \mathrm{rpm}$ on shaking incubator so as to reach the cell population density of $10^{6} \mathrm{per} \mathrm{ml}$.

\subsection{Production of Siderophore}

For production of siderophores, 24 hours old culture of Pseudomonas fluorescens and Pseudomonas aeruginosa were separately inoculated in iron free Succinate medium containing gm/l of K2HPO4 6.0, KH2PO4 3.0, MgSO4 0.2, (NH4)2SO4 1.0 and succinic acid 4.0, pH 7.0, at the constant rate of $1 \% \mathrm{v} / \mathrm{v}$ inoculums and incubated at $28^{\circ} \mathrm{C}$ for $24 \mathrm{hrs}$ with constant shaking at $120 \mathrm{rpm}$ [12]. During incubation, aliquots of media were removed every 2 hours and centrifuged at $5000 \mathrm{rpm}$ for $10 \mathrm{~min}$, the cell free supernatants were subjected to detection

\subsection{Detection of Siderophore}

Siderophore production by both the strains of Pseudomonas spps . was tested qualitatively by Chrome Azural S (CAS) liquid as well as plate assay. The strains were spread over Cetrimide agar and incubated for 48 hrs at $28^{\circ} \mathrm{C}$. After incubation a thin layer of CAS reagent in $0.7 \%$ agar was spread on the bacterial growth and plates were again incubated for $24 \mathrm{hrs}$ at $28^{\circ} \mathrm{C}$, formation of yellow orange color zone around the colonies in plate assay and color change from blue to orange in liquid assay, indicated the siderophore production.

\subsection{Estimation of Siderophore}

The quantitative estimation of siderophore produced by two Pseudomonas spps. was done by CASshuttle assay, in which both the strains were grown on Succinate medium and incubated for $24-30 \mathrm{hrs}$ at $28^{\circ} \mathrm{C}$ with constant shaking at $120 \mathrm{rpm}$ on shaking incubator separately. During incubation, every $20 \mathrm{~min} 5 \mathrm{ml}$ broths were centrifuged at $10,000 \mathrm{rpm}$ at $4^{\circ} \mathrm{C}$ in cooling centrifuge for 10 minute and cell free supernatant was mixed with $0.5 \mathrm{ml}$ CAS solution. The color obtained was measured using the spectrophotometer at $630 \mathrm{~nm}$ with reference containing $0.5 \mathrm{ml}$ uninoculated succinate medium and $0.5 \mathrm{ml}$ CAS solution. The percentage of siderophore units was estimated as the proportion of CAS color shifted using the formula: \% Siderophore units $=[(\mathrm{Ar}-\mathrm{As}) / \mathrm{Ar}] \times 100,(1)$ where $\mathrm{Ar}$ is the absorbance at $630 \mathrm{~nm}$ of reference (CAS assay solution+ uninoculated media) and As is the absorbance at $630 \mathrm{~nm}$ of the sample (CAS assay solution + supernatant) [13].

\subsection{Siderophore Production in Different Media}

For production of siderophores, Cas-amino acid medium, Nutrient broth and Succinic acid medium were separately inoculated with $P$. fluorescens and $P$. aeruginosa and incubated. The siderophore contents were measured by quantitative CAS assay.

\subsection{Characterization of Siderophores}

\subsubsection{Hydroxamate Type of Siderophore}

\subsubsection{Tetrazolium test}

This test is based on the capacity of hydroxamic acids to reduce tetrazolium salt by hydrolysis of hydroxamate groups using a strong alkali. The reduction and the release of alkali shows red color. To a pinch of tetrazolium salt, added 1-2 drops of $2 \mathrm{~N} \mathrm{NaOH}$ and $0.1 \mathrm{ml}$ of the test sample. Instant appearance of a deep red color indicated the presence of hydroxamate siderophore [14].

\subsubsection{Neilands spectrophotometric Assay}

The hydroxamate nature of siderophore was detected by Neilands spectrophotometric assay where a peak between $420-450 \mathrm{~nm}$ on addition of $2 \%$ aqueous solution of $\mathrm{FeCl}_{3}$ to $1 \mathrm{ml}$ of cell-free supernatant indicated the presence of Ferrate hydroxamate [9].

\subsubsection{Catecholate Type of Siderophore}

2.6.2.1 Arnow's Test 
To $1 \mathrm{ml}$ of cell-free supernatant was added $1 \mathrm{ml}$ of $0.5 \mathrm{~N} \mathrm{HCl}$ and $1 \mathrm{ml}$ of nitrite- molybdate reagent, the catechols produced yellow color, immediately $1 \mathrm{ml} \mathrm{NaOH}$ solution was added which resulted in red color formation. The color was stable for 1 hour, and the absorbance was measured at $510 \mathrm{~nm}$ using a UV-vis spectrophotometer.

\subsubsection{Spectrophotometric Assay}

Catecholate siderophores were detected by spectrophotometric assay, where a peak at $495 \mathrm{~nm}$ on addition of $2 \%$ aqueous solution of $\mathrm{FeCl}_{3}$ to $1 \mathrm{ml}$ of cell-free supernatant indicated the presence of catecholate siderophore [15].

\subsection{Effect of $\mathrm{pH}$ on siderophore production}

The effect of $\mathrm{pH}$ between 5.0 to 10.0 on siderophore production was studied in Succinate medium by adjusting the $\mathrm{pH}$ before inoculating the strain with $1 \mathrm{~N} \mathrm{HCl}$ or $1 \mathrm{~N} \mathrm{NaOH}$ and keeping all other conditions constant.

\subsection{Effect of iron on siderophore production}

To determine the effect of iron concentration on the siderophore production, the Succinate medium was supplemented with iron $\left(\mathrm{FeCl}_{3}\right)$ at conc. 1 to $50 \mu \mathrm{M}$ in different sets, for both the Pseudomonas spps. separately. Following the inoculation and incubation at $28^{\circ} \mathrm{C}$ for 24 hours at $120 \mathrm{rpm}$, the siderophore contents were estimated.

\subsection{Influence of amino acids on siderophore production}

To observe the effect of amino acids on siderophore production, the Succinate medium was individually supplemented with $0.1 \mathrm{gm}$ per $100 \mathrm{ml}$ of serine, lysine, alanine, arginine and tyrosine. Each set was separately inoculated with $P$. fluorescens and $P$. aeruginosa and incubated. After incubation of $24 \mathrm{hrs}$ at $28^{\circ} \mathrm{C}$ and $120 \mathrm{rpm}$, each set was subjected to siderophore quantification.

\subsection{Pot culture study}

The Triticum aestivum and Apios americana seeds were surface sterilized by giving alcohol treatment. After drying seeds were soaked in the suspension of $P$. fluorescence and P. aeruginosa separately for few minutes in order to coat them with test organisms then the seeds were removed and allowed to dry. The soil was sterilized by autoclaving and seeds were sowed in soil as a test (inoculated seeds) and control (uninoculated seeds) for both the organisms. Water was added in the pots as per requirement daily and observed for shoot length, root length and seed germination with respect to control after 20 days [1].

\subsection{Detection of siderophore}

\section{Results And Discussion}

Siderophore production by two Pseudomonas spps. was confirmed by color change of CAS reagent from blue to red in qualitative liquid CAS Assay (fig. 1). The color change from blue to orange resulted by siderophoretic removal of $\mathrm{Fe}$ from the dye [16]. The maximum siderophoregenesis was obtained on succinate medium as compared to other media. This was due to pyoverdine, in which the 3-amino moiety of the chromophore is substituted with a group derived from succinate[17].

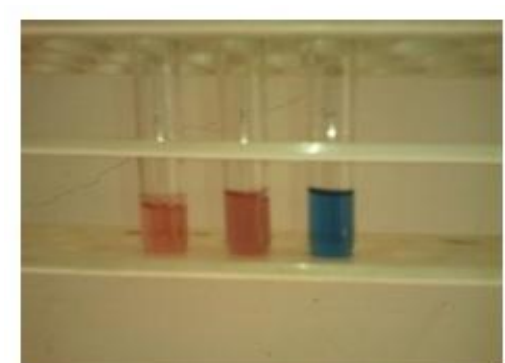

Fig. 1 Color change of CAS reagent from blue to red in qualitative CAS assay.

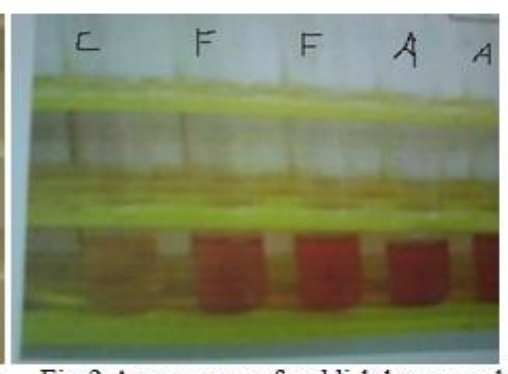

Fig. 2 Appearance of reddish brown color in culture supernatant on addition of $\mathrm{FeCl} 3$ solution

\subsection{Quantitative CAS assay}

In quantitative CAS assay, percent siderophore units were estimated as the proportion of CAS color shifted. It was found that $P$. fluorescens and $P$. aeruginosa produced $88 \%$ and $83 \%$ siderophore units respectively (1). 


\subsection{Characterization of sidrophores}

Appearance of reddish -brown color in $\mathrm{FeCl}_{3}$ test (fig. 2), instant appearance of deep red color in tetrazolium test (fig. 3) and spectrophotometric analysis of the culture in standard succinate medium showed a peak between 420 to $450 \mathrm{~nm}$ (Fig.4) indicated the presence of siderophores of ferrate hydroxamate nature by $P$. fluorescens and P.aeruginosa. Formation of yellow color in Arnow's test and a peak obtained between 490 to $505 \mathrm{~nm}$ (Fig. 5) confirmed the presence of siderophores of catecholate nature by P. aeruginosa [18].

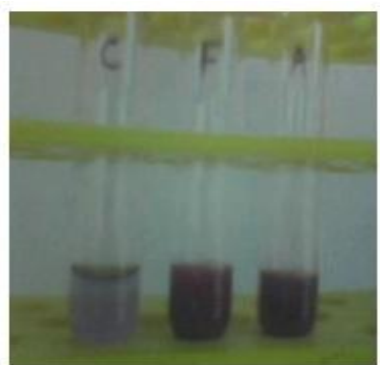

Fig. 3 Instant appearance of deep red color in Tetrazolium test

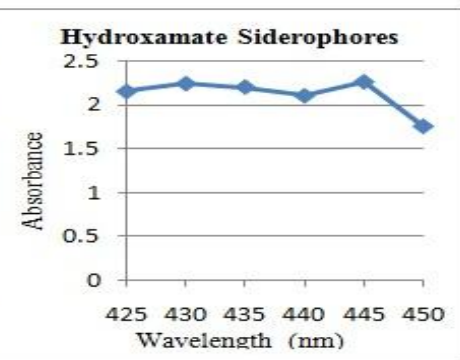

Fig.4 Absorption characteristics of $P$. fluorescens extract confirming hydroxamate nature of siderophore

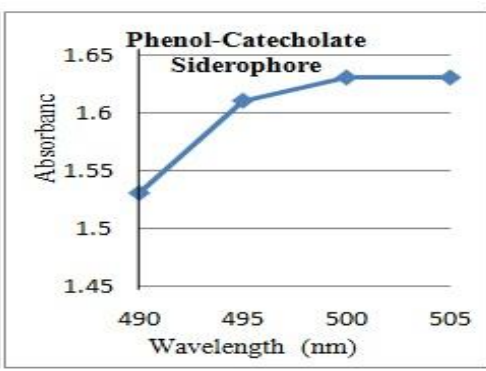

Fig.5 Absorption characteristics of $P$. aeruginosa extract confirming catecholate nature of siderophore

\subsection{Effect of $\mathrm{pH}$ on siderophore production}

The effect of $\mathrm{pH}$ on siderophore production is depicted in fig.6. For both the Pseudomonas spps., the optimum $\mathrm{pH}$ for siderophoregenesis was found to be 7.0, but $P$. fluorescens was more stable to $\mathrm{pH}$ variations as compared to $P$. aeruginosa. Moreover both the organisms had retained at least $50 \%$ of siderophoregenesis activity at all the $\mathrm{pH}$ levels tested.

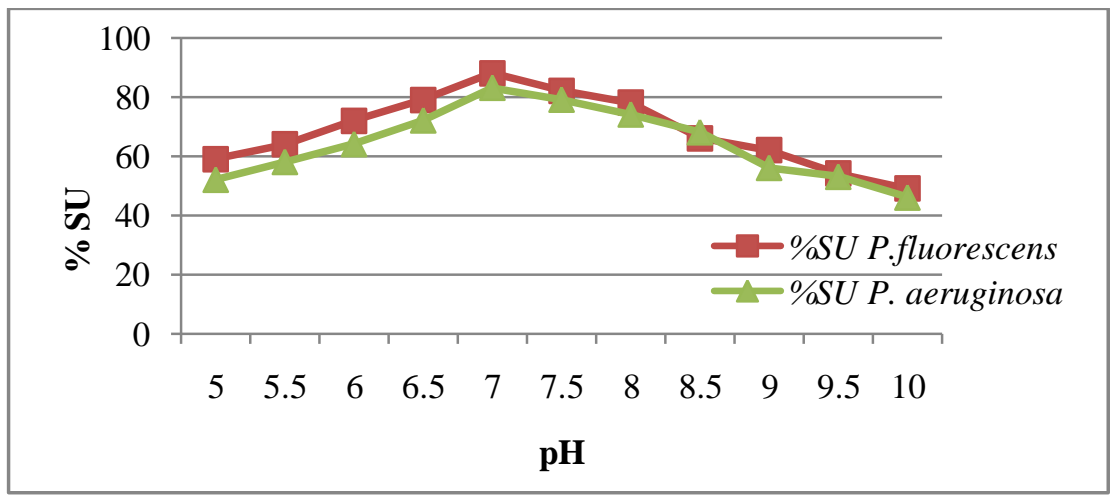

Fig. 6 Effect of $\mathrm{pH}$ on siderophore production in Succinate medium by $P$. fluorescens and $P$. aeruginosa

\subsection{Effect of iron on siderophore production}

The maximum siderophore production was observed in Succinate medium both the Pseudomonas spps. without the addition of iron. The siderophore production was inversely proportional to the iron concentration while no siderophoregenesis was reported at iron concentration above $25 \mu \mathrm{M}$ by $P$. fluorescens and $P$. aeruginosa (fig.7).

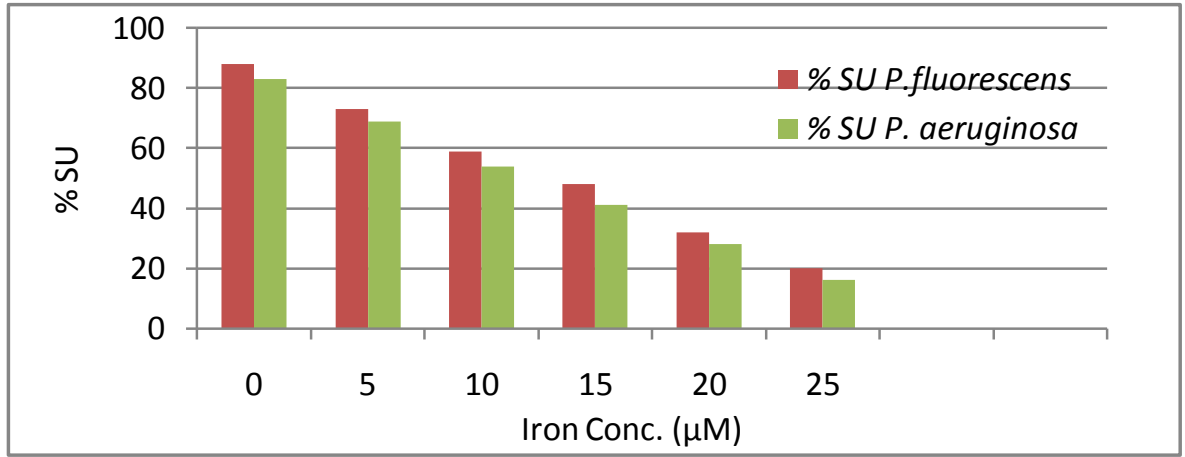

Fig. 7 Effect of iron conc. on siderophore production in Succinate medium by $P$. fluorescens and $P$. aeruginosa. 
Fluorescent Pseudomonads As Plant Growth Promoting Rhizobacteria And Their Siderophoregenesis

\subsection{Effect of amino acids on siderophore production}

All the tested amino acids positively affected the siderophore production. However, threonine resulted in maximum production of $89.02 \%$ and $83.90 \%$ siderophore units for $P$. fluorescens and P. aeruginosa respectively (Table 1).

\begin{tabular}{|l|l|l|l|l|l|l|}
\hline Amino acid & Cys & Thr & Ser & Met & Ala & Lys \\
\hline \% SU P. fluorescens & 88.36 & 89.02 & 88.09 & 89.14 & 88.53 & 88.27 \\
\hline \% SU P. aeruginosa & 83.00 & 83.90 & 83.19 & 83.42 & 83.09 & 83.64 \\
\hline
\end{tabular}

Table.1 Influence of amino acids on siderophore production.

\subsection{Pot Culture Studies}

Influence of Pseudomonas spp. on seed germination and plant growth promotion of Triticum aestivum (fig. 8) and Apios americana (fig. 9) plants are summarized in Table-2 and Table-3 respectively. Both the Pseudomonas spp. had positive stimulatory effect.

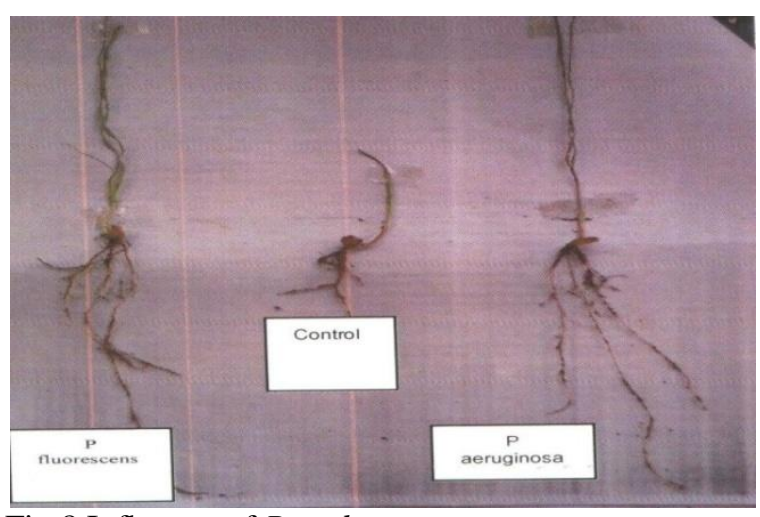

Fig.8 Influence of Pseudomonas spp. on growth promotion of Triticum aestivum plant.

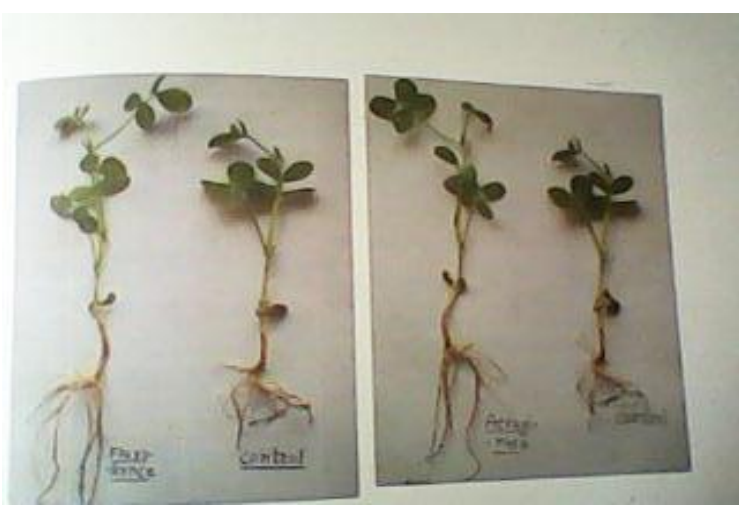

Fig.9 Influence of Pseudomonas spp. on growth promotion of Apios americana plant.

Table-2 Influence of Pseudomonas spp. inoculation on seed germination and growth of Triticum aestivum plant.

\begin{tabular}{|l|l|l|l|l|}
\hline & Organism & Shoot length (cm) & Root length (cm) & Germination (days) \\
\hline Control & - & $6.2 \pm 0.2$ & $4.5 \pm 0.19$ & $6 \pm 0.11$ \\
\hline Test & P. fluorescens & $12.5 \pm 0.12$ & $8.5 \pm 0.63$ & $4 \pm 0.24$ \\
\hline & P. aeruginosa & $11.5 \pm 0.26$ & $7.9 \pm 0.39$ & $4 \pm 0.18$ \\
\hline
\end{tabular}

Table-3 Influence of Pseudomonas spp. inoculation on seed germination and growth of Apios americana plant.

\begin{tabular}{|l|l|l|l|l|}
\hline & Organism & Shoot length $\mathbf{( c m})$ & Root length $(\mathbf{c m})$ & Germination $($ days) \\
\hline Control & - & $6.1 \pm 0.23$ & $3.5 \pm 0.17$ & $7 \pm 0.37$ \\
\hline Test & P. fluorescens & $10.0 \pm 0.62$ & $5.1 \pm 0.27$ & $5 \pm 0.33$ \\
\hline & P. aeruginosa & $9.6 \pm 0.49$ & $4.8 \pm 0.19$ & $7 \pm 0.21$ \\
\hline
\end{tabular}

For Triticum aestivum plant, the promotional efficiency of both the Pseudomonas spps. for shoot length and root length was $76-100 \%$ more and for seed germination was 33\% more as compared to the control. Moreover this potency was apparently higher for $P$. fluorescens than $P$. aeruginosa (fig. 10).

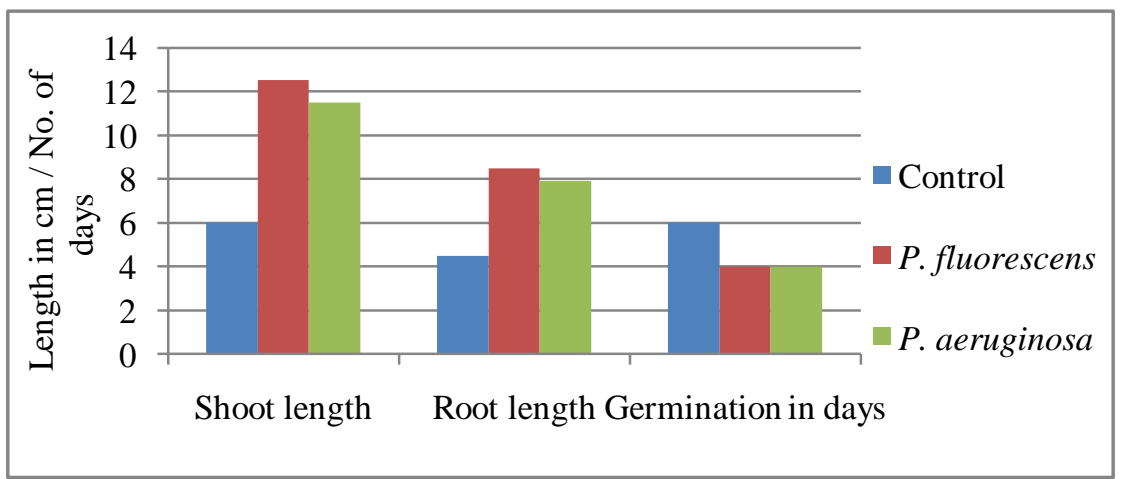

Fig.10. Influence of Pseudomonas spp. inoculation on seed germination and growth of Triticum aestivum plant. 


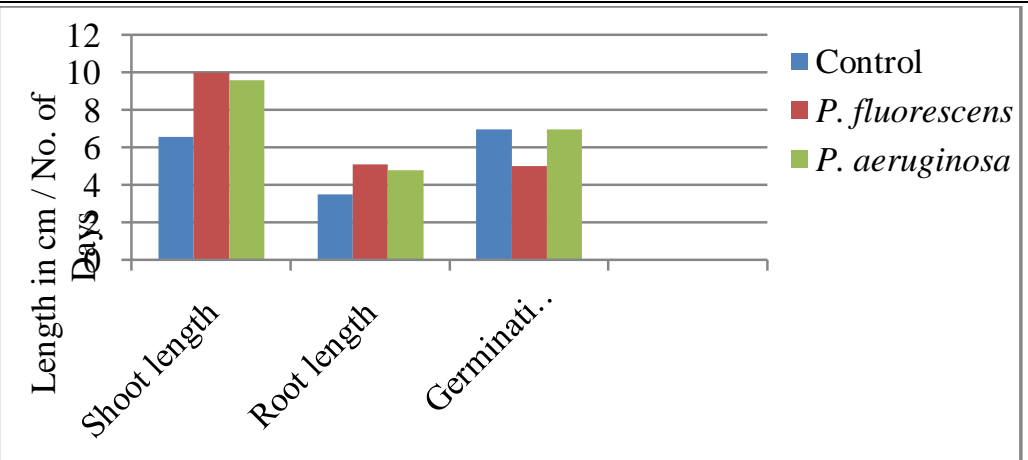

Fig.11. Influence of Pseudomonas spp. inoculation on seed germination and growth of Apios americana plant.

For Apios americana plant, the promotional efficiency of both the Pseudomonas spps. for shoot length and root length was $37-64 \%$ more as compared to the control. Whereas for seed germination only P. fluorescens was $29 \%$ more effective. Overall here also $P$. fluorescens was comparatively more efficient than P. aeruginosa (fig. 11).

\section{Conclusions}

Siderophore production by fluorescent Pseudomonas spps. were confirmed by Universal CAS assay. The color change of CAS from blue to orange-red resulted from siderophoral removal of Fe from the dye. Amount of siderophore produced by both the Pseudomonas spps. were estimated as percentage of siderophore units as the proportion of CAS color shifted. By liquid CAS assay, P. fluorescens and P. aeruginosa have shown the highest yields of siderophore i.e. $88 \%$ and $83 \%$ siderophore units respectively. All tested amino acids positively affected siderophore production. However, threonine resulted in $89.02 \%$ and $83.90 \%$ siderophore units for $P$. fluorescens and $P$. aeruginosa. Further, the type of siderophore was determined. $P$. fluorescens showed formation of hydroxamate type whereas $P$. aeruginosa showed both the types of siderophore production i.e. Hydroxamate (pyoverdine) and phenolate-catecholate type (pyochelin). The maximum siderophoregenesis was obtained on succinate medium as compared to other media tested. This was due to pyoverdine, in which the 3- amino moiety of the chromophore is substituted with a group derived from succinate. The optimum $\mathrm{pH}$ for siderophore production was found to be 7.0 at which bacteria grew better and also iron was present in insoluble form and therefore was not available to the bacteria. Siderophores are iron-specific compounds which are secreted under low iron stress conditions. In the succinate medium, the maximum siderophore production was found by both the Pseudomonas spps. without the addition of iron while its productivity was repressed above iron concentration of $25 \mu \mathrm{M}$. Both the Pseudomonas spps. were found to be very effective in plant growth promotion and seed germination of Triticum aestivum and Apios americana plants.

\section{References}

[1]. R.Z. Sayyed, M. D. Badgujar, H.M. Sonawane, M.M. Mhaske and S.B. Chincholkar, Production of microbial iron chelators (Siderophores) by Fluorescent Pseudomonads, Indian journal of Biotechnology, 4, 2005, 484-490.

[2]. R Decheng, R. Zuo, Thomas and K. Wood, Appl Microbiol Biotechnol. 66, 2005, 689-695.

[3]. M.H. Wilhelmina, J.P. Adam, P.J. Michael., R Jordi, R. Alan and G.M. Alastair, J Clinical Microbiology. 6, $2004,2806-2809$.

[4]. G. Briskot, K. Taraz, and H. Budzikiewicz, Pyoverdine type siderophores from Pseudomonas aeruginosa. Z. Naturforsch. Sect. C, 41, 1986, 497-506.

[5]. C. D. Cox, and P. Adams, Siderophore activity of pyoverdin for Pseudomonas aeruginosa, Infect. Immun. 48, 1985, 130-138.

[6]. S. Wendenbaum, , P. Demange, A. Dell, J. M. Meyer, and M. A. Abdallah, The structure of pyoverdin Pa, the siderophore of seudomonas aeruginosa, Tetrahedron Lett. 24, 1983, 4877-4880.

[7]. S. Diego, M. Hofte, Iron chelation in plants and soil microorganisms (Barton, L.L. and Hemming, B.C. (eds.), Academic Press 1993, 3-6.

[8]. B. Schwyn and J. B. Neilands, Universal chemical assay for the detection and determination of siderophores, Anal Biochem, 160, $1987,47-56$

[9]. J. M. Meyer, D. Honhadel, A. Khan, and P. Cornelis, Pyoverdin-facilitated iron uptake in Pseudomonas Aeruginosa: immunological characterization of the ferripyoverdin receptor. Mol. Microbiol, 4, 1990, 1401-1405

[10]. C. D. Cox, K. L. Rinehart, M. L. Moore, and J. C. Cook, Pyochelin: novel structure of an iron-chelating growth promoter from Pseudomonas aeruginosa, Proc. $78^{\text {th }}$, Natl. Acad. Sci., USA, 1981, 302-308.

[11]. C. D. Cox, and R. Graham, Isolation of an iron-binding compound from Pseudomonas aeruginosa. J. Bacteriol, 137, 1979, 357-364.

[12]. J. M. Meyer, and M. A. Abdallah, The fluorescent pigment of Pseudomonas fluorescens, Biosynthesis, purification and physicochemical properties, J Gen Microbiol. 107, 1978, 319-328.

[13]. S. M. Payne Detection, isolation and characterization of siderophores, Methods Enzymol, 235, $1994,329$.

[14]. B. P. Dave and H.C. Dube, Chemical characterization of fungal siderophores, Indian journal of Experimental Biology, 2000, 38, 5662.

[15. M. A. F Jalal and H. D.Vander, Isolation and spectroscopic identification of fungal siderophores. In: Handbook of microbial iron chelates, Winklemann, G. (ed.), Pegamon Press, 1990 Oxford, 235-269

[16]. M. H. Wilhelmina, A.J. Potter, M.P. Jennings, R. Jordi, A.R. Hauser and A.G. McEwan,

J. Clinical Micriobiology. 42, 2004, 2806-2809.

[17]. C. Linget, D.G. Slylianou, A. Dell, R.E. Wolff, Y. Piemont, and M.A. Abdallah, Tetrahedron Letters, 33, $1992,3851-385$.

[18]. S. S. Ali and N.N Vidhale, Evaluation of Siderophore produced by Different clinical Isolate Pseudomona Aeruginosa, International Journal of Microbiology Research, 3(3), 2011, 131-135. 